\title{
OPEN Identification of a non-coding RNA and its putative involvement in the regulation of tetanus toxin synthesis in Clostridium tetani
}

\author{
Holger Brüggemann ${ }^{1,4}$, Diana Chapeton-Montes ${ }^{2,4}$, Lucile Plourde ${ }^{3} \&$ Michel R. Popoff $0^{2 \bowtie}$
}

Clostridium tetani produces the tetanus toxin (TeNT), one of the most powerful bacterial toxins known to humankind and responsible for tetanus. The regulation of toxin expression is complex and involves the alternative sigma factor TetR as well as other regulators. Here, a transcriptional analysis of the TeNT-encoding large plasmid of $C$. tetani identified a putative non-coding small RNA (sRNA), located in close vicinity of the $3^{\prime}$ untranslated region of the tent gene. A northern blot experiment could identify a respective sRNA with a size of approx. 140 nucleotides. Sequence analysis showed that the sRNA contains a 14-nucleotide region that is complementary to a $5^{\prime}$ located region of tent. In order to investigate the function of the SRNA, we applied a RNA interference approach targeting the sRNA in two $C$. tetani wild-type strains; the constructed antisense $C$. tetani strains showed an approx. threefold increase in both extracellular and total TeNT production compared to the respective wild-type strains. In addition, recombinant $C$. tetani strains were constructed that contained tentlocus harboring plasmids with and without the sRNA. However, the introduction of the tent-locus without the SRNA in a C. tetani strain lacking the wild-type TeNT-encoding large plasmid resulted in a lower TeNT production compared to the same strain with recombinant tent-locus with the sRNA. This suggests that the expression or the effect of the sRNA is modulated by the $C$. tetani genetic background, notably that of the wild-type TeNT-encoding large plasmid. In addition, some recombinant strains exhibited modulated growth patterns, characterized by premature bacterial cell lysis. Taken together, our data indicate that the sRNA acts as a negative regulator of TeNT synthesis, with a possible impact on the growth of $C$. tetani. We hypothesize that the role of this SRNA is to limit toxin levels in the exponential growth phase in order to prevent premature bacterial lysis.

Clostridium tetani is a Gram-positive anaerobic spore-forming bacterium that is widely distributed in the environment. Clostridium tetani produces the tetanus toxin (TeNT) that is responsible for tetanus. The mode of action of TeNT has been investigated since several decades. TeNT produced in a necrotic wound contaminated with $C$. tetani, binds to peripheral nerve termini by interacting with specific receptors including polysialogangliosides and nidogens, then undergoes a retrograde transport in motorneurons and enters inhibitory interneurons in the central nervous system, where it cleaves the SNARE protein VAMP resulting in the blockade of neurotransmitter (glycine and GABA) release. The clinical symptoms are characterized by muscular rigidity, painful spasms and autonomic instability ${ }^{1-4}$.

In C. tetani strain E88, the tent gene is located on a large $74 \mathrm{~kb}$ plasmid, $\mathrm{pE} 88^{5}$. The gene tet $R$ is conserved just upstream of tent, and encodes for an alternative sigma $(\sigma)$-factor, which positively regulates the transcription of tent ${ }^{6}$. Furthermore, the $74 \mathrm{~kb}$ C. tetani plasmid harbors other putative regulatory genes of TeNT synthesis, such as a two-component system (CTP21/CTP22), CTP4 and CTP5 (homologs of Clostridium perfringens UviAB that positively control a UV-inducible bacteriocin gene), CTP10 (a putative $\sigma$-factor/DNA binding protein), and CTP11 (a putative $\sigma$-factor/DNA binding protein) $)^{5}$. At least, three two-component systems and the master regulator of metabolism, CodY, control TeNT synthesis ${ }^{7}$. However, the whole regulatory network controlling TeNT production still remains poorly understood.

\footnotetext{
${ }^{1}$ Department of Biomedicine, Aarhus University, Aarhus, Denmark. ${ }^{2}$ Bacterial Toxins, Institut Pasteur, 25 rue du Dr Roux, 75724 Paris Cedex15, France. ${ }^{3}$ Sanofi-Pasteur, Marcy l'Etoile, France. ${ }^{4}$ These authors contributed equally: Holger Bruggemannand Diana Chapeton-Montes. ${ }^{\circledR}$ email: popoff2m@gmail.com
} 


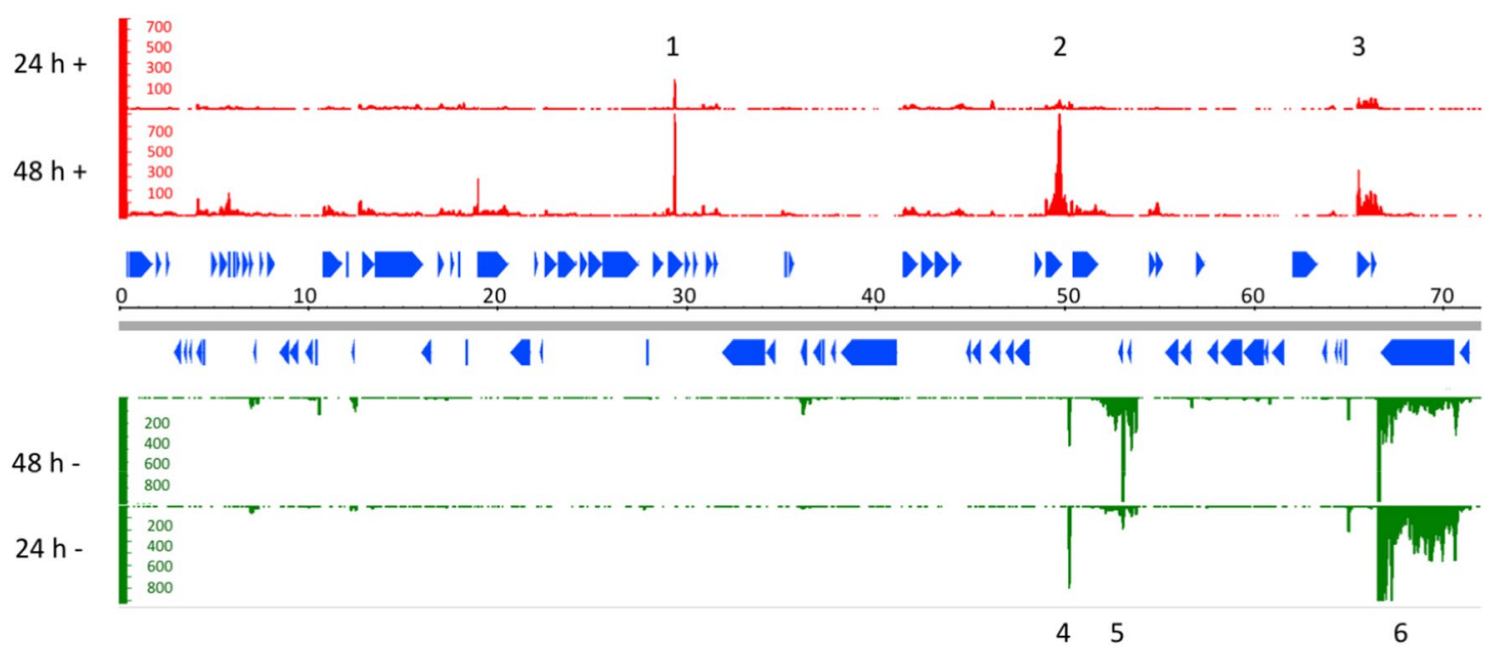

Figure 1. Transcriptome of the tent-containing $74 \mathrm{~kb}$ plasmid in C. tetani strain A when grown under anaerobic conditions in TGY medium for 24 and $48 \mathrm{~h}$. RNA-seq data was mapped to the plus and minus strand of the $74 \mathrm{~kb}$ reference plasmid; the nucleotide position is given in $\mathrm{kb}$ (grey bar). The blue triangles indicate coding sequences. Peaks of high transcription are labelled: 1, CTP27 (encoding a putative 23S rRNA (guanine-N1) methyltransferase); 2, CTP44 (gene of unknown function); 3, CTP58/CTP59 (genes of unknown function); 4, intergenic region between CTP44 and CTP45 (the latter encodes a putative replication protein); 5, CTP46 and CTP47 (genes of unknown function); 6, tent gene (CTP60). The image was created with the Integrative Genome Browser (IGB v8.5.4; https://bioviz.org/).

The importance of regulation by small regulatory RNAs (sRNAs) has been recognized in prokaryotes ${ }^{8,9}$. sRNAs are key players in mediating bacterial responses to environmental signals. In addition, they are important regulators of virulence in several pathogenic bacteria. Furthermore, the regulation by sRNAs is advantageous when fast responses to external signals are needed ${ }^{10,11}$. Sizes of bacterial sRNA are typically between 50 and 500 nucleotides. Regulatory sRNAs have been identified in different genomic localizations. They can be found in or overlapping with $5^{\prime}$ and $3^{\prime}$ untranslated regions (UTRs) next to the coding sequence, in intergenic regions, and as antisense RNAs transcribed on the opposite strand of the open reading frame $(\mathrm{ORF})^{11,12}$. In the majority of cases, antisense RNA results in post-transcriptional inhibition of target mRNA function, but in a few cases, activating mechanisms have been involved ${ }^{8,13}$. Their structures are more stable than those of mRNAs. They begin with a sequence that can fold into a stable stem-loop, and transcription terminates with a rho-independent transcription terminator, a stem-loop that also helps to stabilize the molecule ${ }^{14,15}$.

Regulatory RNAs are divided into (1) cis-acting antisense RNAs, which are transcribed from the DNA strand opposite to the target mRNA, and display perfect base complementarities to their targets, and (2) trans-acting antisense RNAs, which are transcribed from distant loci from target mRNAs, and are only partially complementary with their mRNA targets. The RNA chaperone Hfq protein (in Gram-negative bacteria) helps target recognition to promote trans-antisense RNA binding by an unknown mechanism ${ }^{11}$. Regulatory RNAs are the most abundant class of post-transcriptional regulators. Small non-coding RNAs control their targets through different mechanisms, including: (1) translational inhibition, which can occur by direct blocking of the ribosomebinding site (RBS) or by induction of structural alterations downstream of the RBS; (2) alteration of mRNA stability, whereby both cis- and trans-acting RNAs can promote RNA degradation; (3) protein sequestration, via regulatory RNA/protein interaction, thus inducing numerous downstream effects; (4) interaction with DNA (CRISPR-RNA, Clustered Regularly Interspaced Short Palindromic Repeats-RNA) and (5) riboswitches, i.e. regulatory RNAs that bind metabolites or environmental cues ${ }^{8,13}$.

Regulatory sRNAs have been identified in clostridia ${ }^{16-19}$. In C. perfringens, a small RNA named VR-RNA (VirR regulated RNA) controls the expression of the alpha- (plc), kappa- (colA) and beta2-toxin genes in a VirR/ VirS dependent manner ${ }^{18,20}$. Another regulatory RNA, VirX, controls the levels of theta-toxin ( $\left.p f o A\right), p l c$, and colA mRNAs independently of the VirR/VirS regulatory cascade ${ }^{21}$.

The goal of this study was to identify and investigate putative regulatory RNAs in the vicinity of tent in $C$. tetani and decipher their role in tetanus toxin synthesis. Our results support the identification of a novel sRNA overlapping with the $3^{\prime} \mathrm{UTR}$ of tent, that putatively functions as a negative regulator of TeNT synthesis.

\section{Results}

Transcriptional profile of the tent-containing large plasmid. We first recorded the transcriptome of the tent-containing $74 \mathrm{~kb}$ plasmid in C. tetani strain A when grown under anaerobic conditions in TGY medium for 24 and $48 \mathrm{~h}$ (Fig. 1). A few plasmid regions were highly transcribed. On the plus strand, there were strong transcription peaks within genes CTP27 encoding a putative 23S rRNA (guanine-N1-methyltransferase), CTP44 (protein of unknown function) and CTP58/CTP59 (proteins of unknown function). On the minus strand, strongest transcription was associated with an intergenic region between CTP44 and CTP45, the latter encodes a putative replication protein, and with the genes CTP46 and CTP47 (proteins of unknown function). By far the strongest transcription was associated with tent (CTP60), in particular at $24 \mathrm{~h}$ of growth. Regarding 


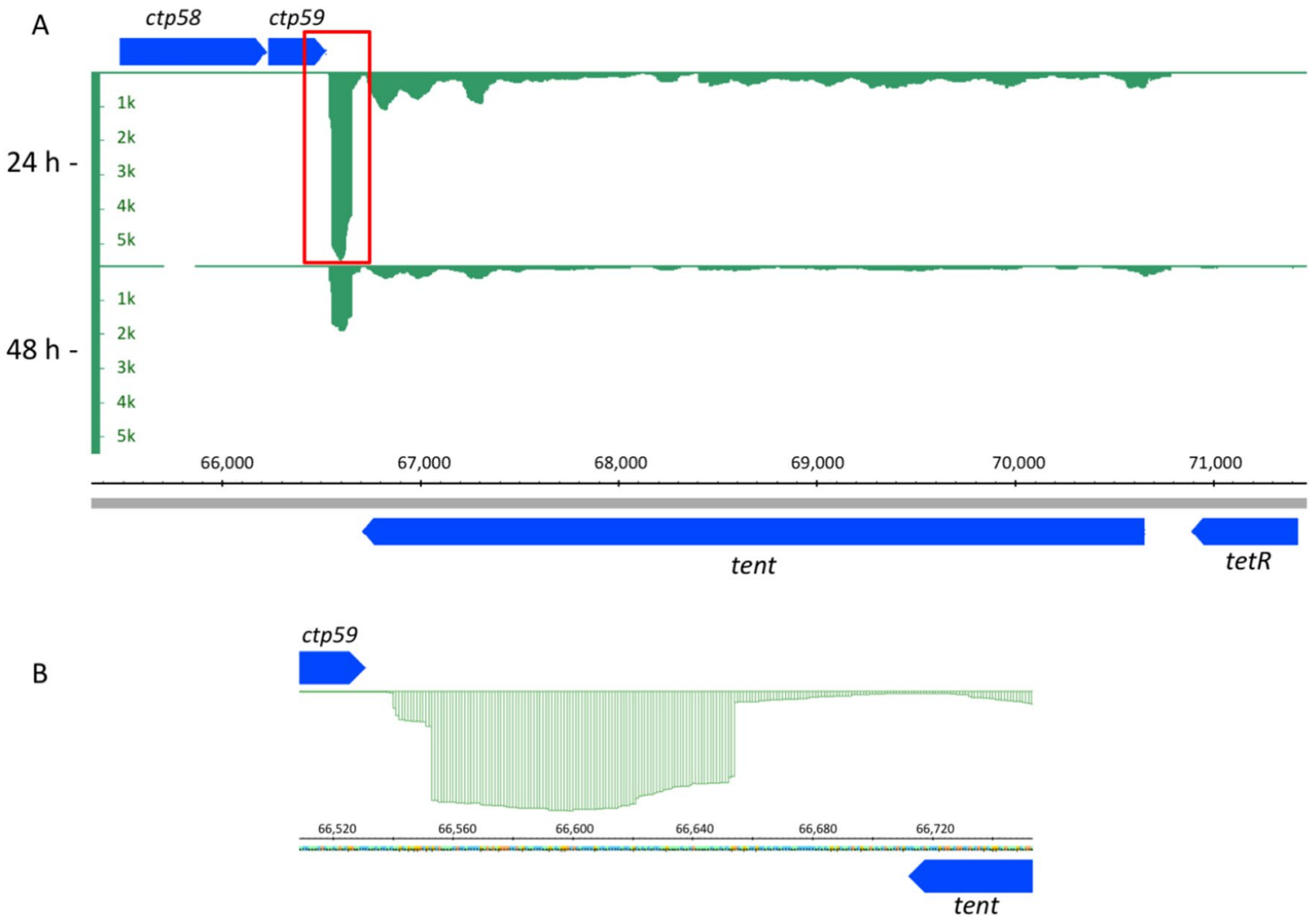

Figure 2. Transcriptional profile of a sRNA downstream of tent. (A) A plasmid region located downstream of the protein-coding part of tent was highly transcribed in C. tetani grown for $24 \mathrm{~h}$ in TGY medium. (B) An enlarged cutout from 2A (red box) illustrates the exact region of the putative sRNA. CDS are indicated in blue. The nucleotide position of the $74 \mathrm{~kb}$ plasmid is given in bp (grey bar). The image was created with the Integrative Genome Browser (IGB v8.5.4; https://bioviz.org/).

other putative regulatory genes located on the plasmid, we noticed very weak transcription of the two genes that encode putative RNA polymerase sigma factors (CTP10, CTP11), and also weak expression of the putative twocomponent system (CTP21/CTP22).

A 3'UTR region of tent is transcribed in C. tetani. A closer inspection of the transcriptional profile of tent revealed a strong transcriptional peak on the minus strand, located downstream of the protein-encoding part of tent and downstream of the ctp59 gene located on the plus strand (Fig. 2). The transcriptional peak of $114 \mathrm{nt}$ (exact coordinates 66,540-66,654 nt of the plasmid) seems to be located in, or overlapping with the 3'UTR region of tent, since transcription continued downstream of the protein-encoding part of tent. In order to exclude the possibility that this peak represents a mRNA from a small peptide-encoding gene, we performed a bioinformatics analysis of the six ORFs in the area between tent and ctp59, but could not detect any likely candidates for peptide-encoding CDS overlapping with the region of the transcriptional peak. We then hypothesized that this peak corresponds to a putative non-coding sRNA. Its location in close vicinity of tent could indicate that it is involved in controlling TeNT synthesis.

The $114 \mathrm{nt}$ sequence of the putative sRNA was identified also in all other plasmid-positive Harvard-derived strains clade $1 \mathrm{~A}$ strains, according to ${ }^{22}$, such as E88, CN655, and strain A with a $100 \%$ identity on nucleotide level. In addition, all plasmid-positive $C$. tetani strains of clades $1 \mathrm{~B}$ to $1 \mathrm{E}$ carry an identical sequence (with strain TMB2, a clade 1B strain, as the only exception) (Sup. Fig. S1). In the other C. tetani strains, i.e. strains of clades $1 \mathrm{~F}$ to $1 \mathrm{H}$ and clade 2 , the sequence is present with four SNPs. We used the Rfam database (a collection of sRNA families) to search whether the putative sRNA has homology to known sRNAs ${ }^{23}$, but no hit was found.

According to RNA folding predictions, the putative sRNA of clade 1 strains forms two stem-loop structures with one big interior junction-loop (Fig. 3A). In clade 2 and clade $1 \mathrm{G}$ strains, the RNA folds differently, with an additional loop within the interior junction-loop (Fig. 3B). The stem-loop that is most distant from tent contains a Rho-independent terminator as judged from an ARNold analysis ${ }^{24,25}$. This is in agreement with other sRNAs, as transcription of most sRNA genes is terminated by Rho-independent termination ${ }^{14}$.

We hypothesize that the secondary structure could potentially sequester tent. The IntaRNA tool was used to search for a potential target of this sRNA. The most significant match was found between the interior junctionloop-exposed sequence (between position 47 and 61) of the putative sRNA and a region in the 5'end coding sequence of tent (Fig. 3C). This could indicate a direct interaction of the putative sRNA with the tent mRNA. 
A

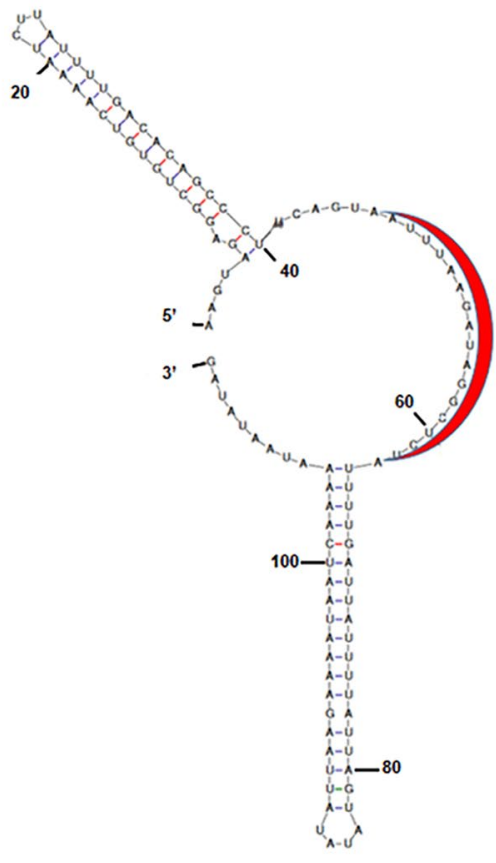

B

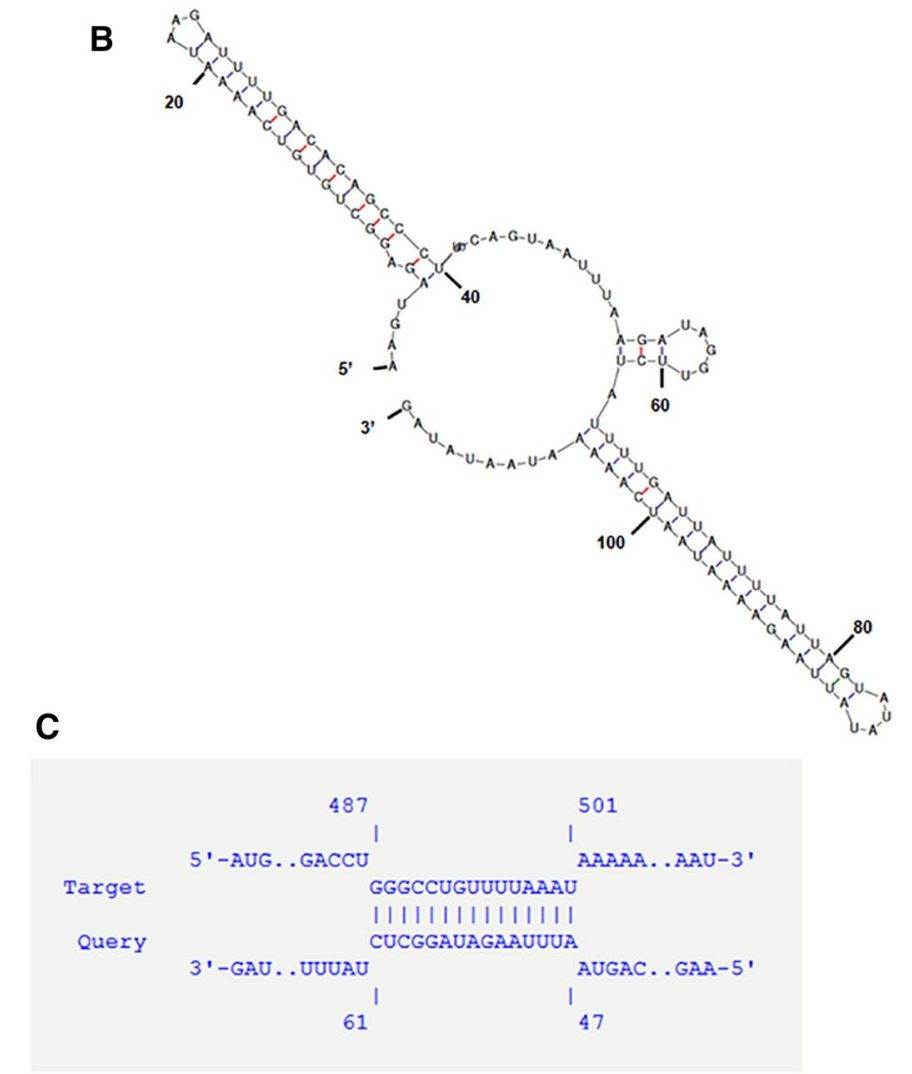

C

Figure 3. Predicted secondary structure of the putative sRNA of C. tetani and its potential binding site. (A) Mfold prediction of the sRNA in most $C$. tetani clade 1 strains, including the Harvard derived strains. The red arc represents the potential binding site with tent. The stem-loop that is most distant from tent contains a Rho-independent terminator, according to an ARNold analysis. (B) Mfold prediction of the sRNA from $C$. tetani clade 2 and $1 \mathrm{G}$ strains. (C) A nearly perfect $14 \mathrm{nt}$ match of the interior junction-loop-exposed sequence (nucleotide positions 47-61) of the sRNA with the 5' end of tent mRNA (nucleotide positions 487-501 of tent) was detected. The image was created with mfold (v3.5; http://www.unafold.org/).

Expression of the putative sRNA downstream of tent. The existence and expression of the putative sRNA was investigated using Northern blot analysis and qRT-PCR, respectively. Northern blot analysis could identify the sRNA as an abundant RNA molecule of approx. $140 \mathrm{nt}$, which is in accordance with the predicted size deduced from RNA-seq data (Fig. 4A). An additional less abundant longer transcript (approx. $170 \mathrm{nt}$ ) was also detected and, albeit to a much lower extent, other longer transcripts in a scaled partition that could correspond to multimers. Expression of the sRNA by qRT-PCR was determined in C. tetani CN655 grown in TGY media for $24 \mathrm{~h}$ and $48 \mathrm{~h}$. This analysis revealed that the sRNA was abundantly expressed, but only at early time points (Fig. 4B). RNA-seq confirmed a weaker transcription of the sRNA at $48 \mathrm{~h}$ compared to $24 \mathrm{~h}$ of culture (Fig. 2).

The SRNA modulates tetanus toxin production in C. tetani. In order to investigate the regulatory function of the sRNA on toxin synthesis, we constructed a sRNA antisense strain, using an RNA antisense system that has previously been employed in Clostridium botulinum and C. tetani ${ }^{6,26}$. The plasmid construct with the antisense sRNA was named p1421 (Sup. Fig. S2). The construct was transformed into the wild-type strain CN655, a TeNT-producing clade 1A strain. The recombinant strain targeting the sRNA (CN655/p1421) showed a strong significant increase in both extracellular and total TeNT production at most time points of the exponential and stationary growth phases (Fig. 5). At $24 \mathrm{~h}$, extracellular TeNT levels were threefold increased in the CN655/p1421 strain compared to the control strain CN655/pAT18 (Fig. 5B). In addition, fivefold increased tent transcription in the early growth phase $(8 \mathrm{~h})$ was observed in strain CN655/p1421 compared to the control strain CN655/pAT18 (Fig. 5D). It is noteworthy that the changes in TeNT synthesis were of a larger extend than those in tent mRNA levels, suggesting additional post-transcriptional regulation. Taken together, this indicates that the sRNA acts as a negative regulator in C. tetani in the early growth phase, leading to decreased TeNT production.

The RNA antisense system targeting the sRNA was also tested in another Harvard derivative clade 1A strain, C. tetani strain A (Fig. 6). This strain produces high amounts of TeNT (three- to fivefold more TeNT production than CN655 in TGY culture, data not shown) and its growth profile is characterized by a rapid growth until approx. $24 \mathrm{~h}$, followed by substantial bacterial lysis from 24 to $56 \mathrm{~h}$ and then by a stationary phase $(56-144 \mathrm{~h})$ (Fig. 6A). Similar to strain CN655/p1421 (Fig. 5), extracellular TeNT levels and total TeNT production were increased in strain A/p1421 cultures compared to the control strain A/pAT18 (Fig. 6B,C). However in the strain 

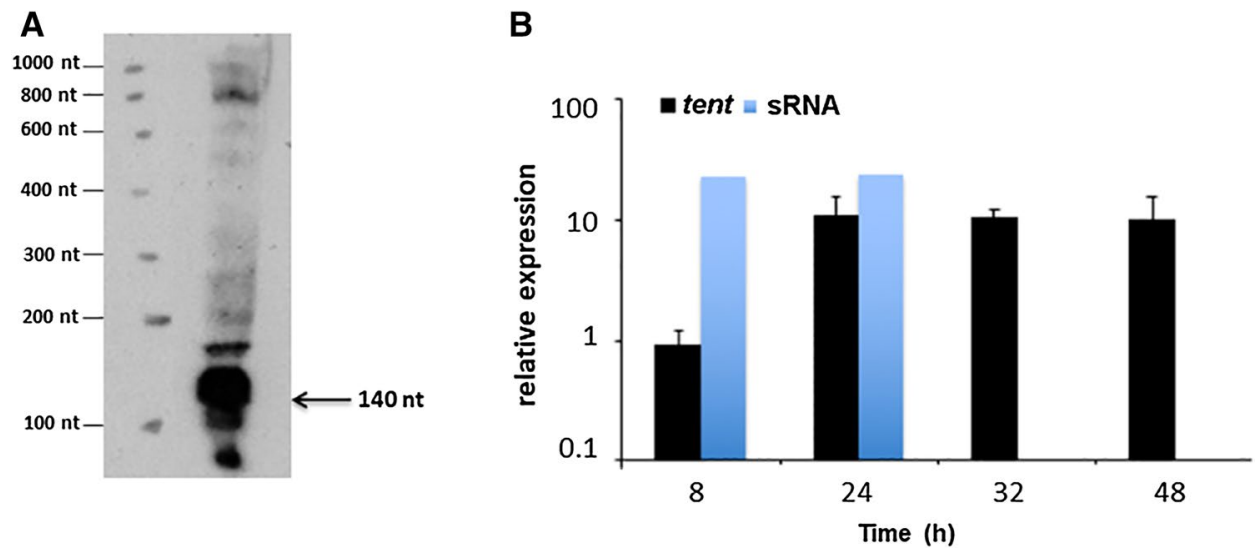

Figure 4. Detection of sRNA by Northern blot in C. tetani and its relative expression. (A) Northern blot analysis. The arrow points to the detected transcript with its size estimated by comparison with RNA molecular weight standards. Total RNA was extracted from C. tetani CN655 grown $24 \mathrm{~h}$ in TGY. sRNA was detected in Northern blot with biotinylated P2275 (TAATCAAAATAGAGCCTATC). (B) Relative expression in logarithmic scale of tent and sRNA in C. tetani strain CN655 grown in TGY for the indicated times.

A
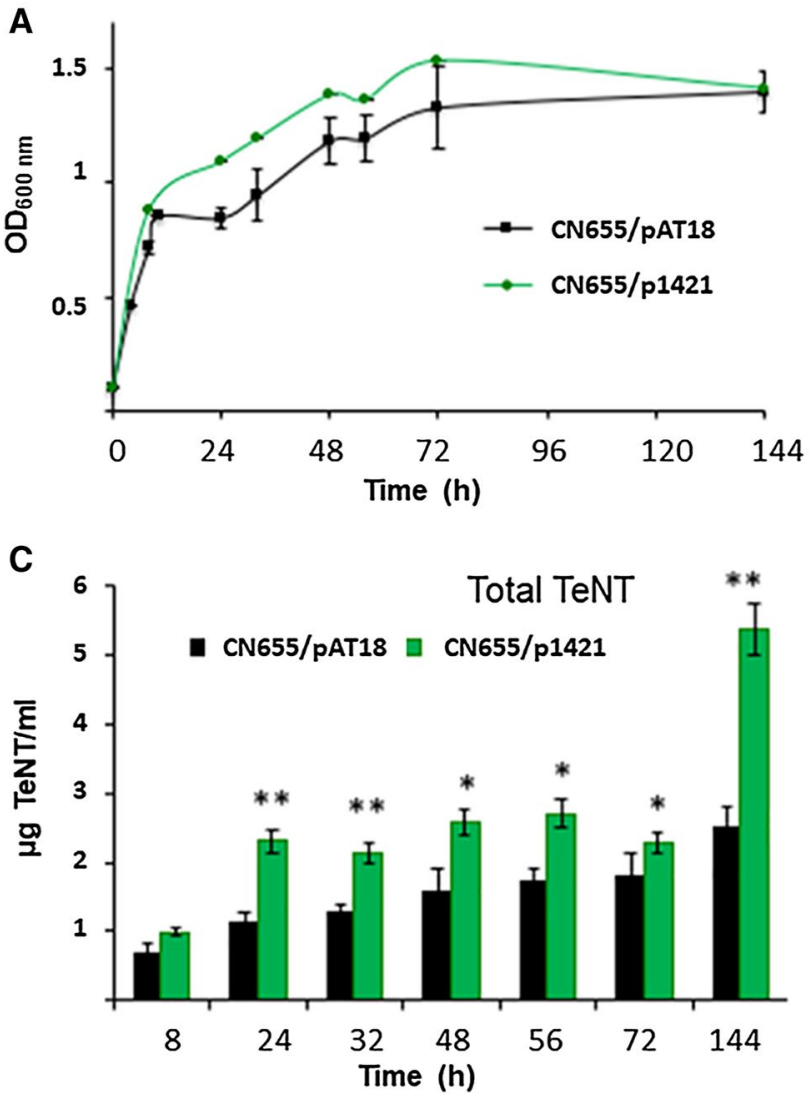

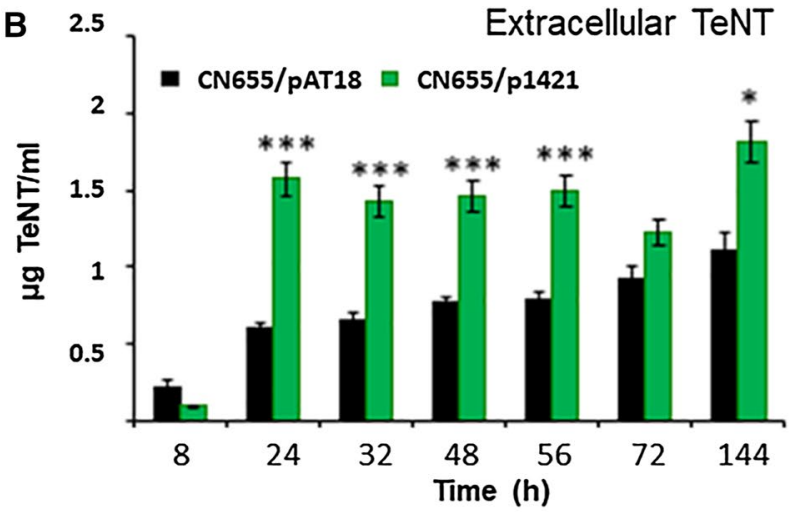

D

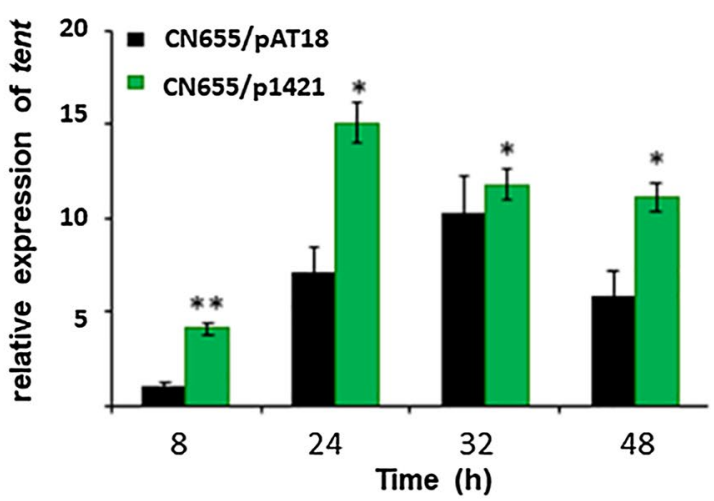

Figure 5. Effect of introducing an antisense sRNA construct (p1421) on growth, TeNT production, and tent expression in C. tetani CN655. (A) Growth kinetics in TGY medium of the recombinant CN655/p1421 (antisense sRNA construct) strain was similar to that of the control strain CN655/pAT18 (control vector). (B) Extracellular TeNT was increased in the culture supernatant of CN655/p1421 (antisense sRNA construct) compared to CN655/pAT18 (control vector). (C) Total TeNT production was increased in CN655/p1421 (antisense sRNA construct) compared to CN655/pAT18 (control vector). (D) Expression of tent was increased in CN655/p1421 (antisense sRNA construct) compared to CN655/pAT18 (control vector). Target gene expression was normalized to $r p o B$ and $g y r A$. Statistical significance of differences between the control strain and recombinant strains is indicated with $p$-values $\left({ }^{\star} P<0.05\right.$; ${ }^{*} P<0.01$; $\left.{ }^{\star *} P<0.001\right)$. The data are from three independent experiments. 
A

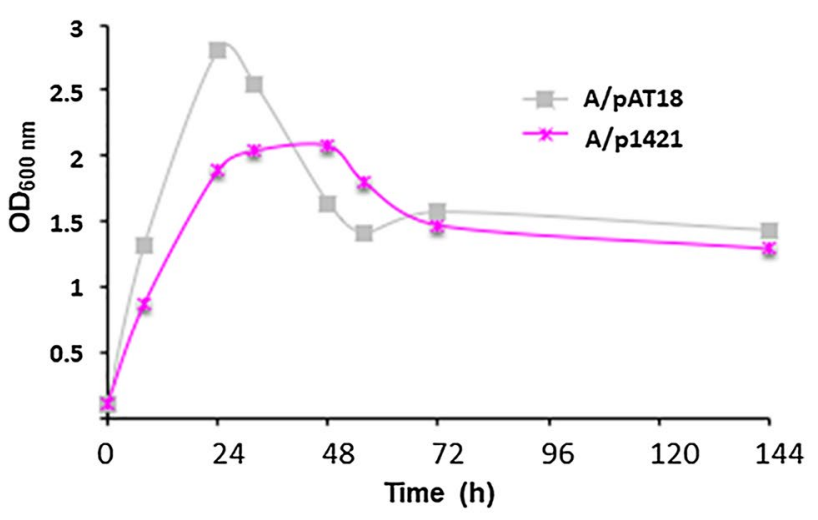

B Extracellular TeNT

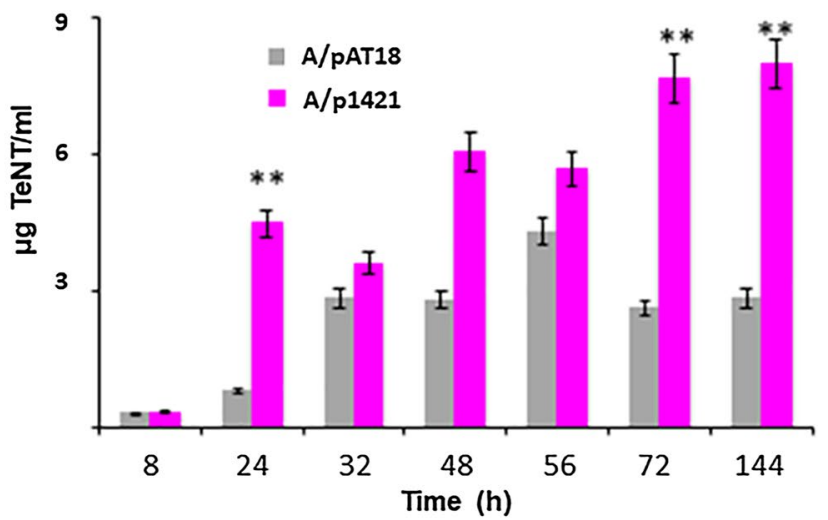

C
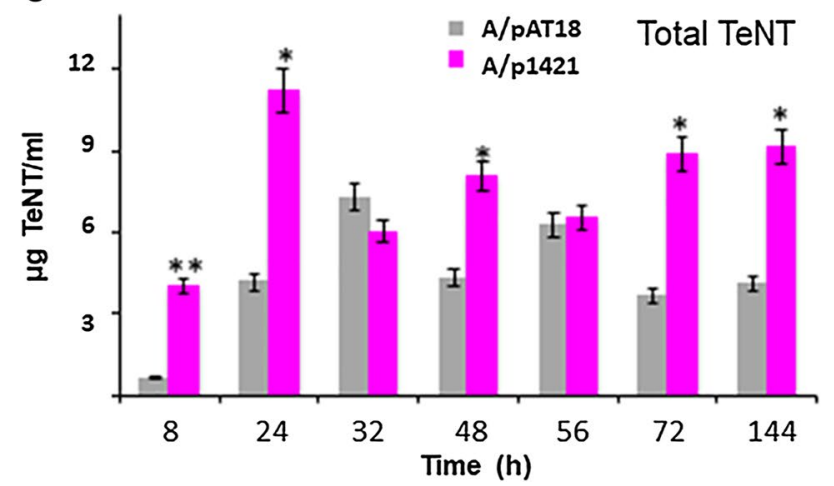

Figure 6. Effect of introducing an antisense sRNA construct (p1421) on growth, TeNT production, and tent expression in C. tetani strain A. (A) Growth kinetics of the recombinant strain A/p1421 (antisense sRNA construct) showed a slower growth and a reduced bacterial lysis after $24 \mathrm{~h}$ of culture in TGY medium compared to the control strain A/pAT18. (B) Extracellular TeNT was significantly increased in strain A/p1421 compared to the control strain A/pAT18. (C) Total TeNT production was increased in strain A/p1421 compared to the control strain A/pAT18. Statistical significance of differences between the control strain and recombinant strains is indicated with $\mathrm{p}$-values $\left({ }^{\star} P<0.05 ;{ }^{*} P<0.01\right)$. The data are from three independent experiments.

A, the exponential growth and bacterial lysis after $48 \mathrm{~h}$ of culture were less pronounced in the recombinant strain A/p1421 than in the control A/pAT18 (Fig. 6A).

Expression of recombinant tent loci with and without sRNA in C. tetani. To further investigate the influence of the sRNA in C. tetani, the region corresponding to tetR-tent with their own promoters and the sRNA, and tetR-tent with their own promoters but lacking the sRNA were cloned into the shuttle vector pAT18, yielding p1423 and p1424, respectively (Sup. Fig. S2). We took advantage of the strain 1586-Z1, which is a non-toxigenic derivative of the clade $1 \mathrm{~A}$ strain 1586-U122. Both strains, 1586-Z1 and 1586-U1, retain the same chromosomal genome sequence, but 1586-Z1 lacks the wild-type large plasmid harboring tent ${ }^{22}$. The recombinant plasmids p1423 and p1424 were transformed into 1586-Z1 to compare the expression of the recombinant tetR-tent genes, with and without the putative sRNA in a C. tetani genomic background.

TeNT production was observed in 1586-Z1 transformed with p1423 or p1424. High levels of extracellular TeNT were measured in 1586-Z1 when p1423 containing functional copies of tetR, tent and sRNA (Fig. 7B). Extracellular TeNT levels in 1586-Z1/p1423 were in a similar range than in CN655/p1421 (Fig. 5B). Unexpectedly, 1586-Z1/p1424 (containing functional copies of tetR, tent but lacking the sRNA) showed lower levels of extracellular TeNT in the late growth phase (32-144 h) compared to strain 1586-Z1/p1423 (Fig. 7B). However, the strain 1586-U1 (which contains the wild-type TeNT-encoding large plasmid), transformed with p1423 and p1424 showed a different result. In contrast to 1586-Z1, the extracellular TeNT production was higher in 1586$\mathrm{U} 1 / \mathrm{p} 1424$ (containing functional copies of tetR, tent but lacking the sRNA) than in $1586-\mathrm{U} 1 / \mathrm{p} 1423$ (containing functional copies of tetR, tent and sRNA) (Sup. Fig. S3), thus exhibiting a similar pattern than strain A/p1421 (Fig. 6B). The apparently discrepant observation might result from different copy number ratios of tetR-tent genes and sRNA in strains with and without the wild-type TeNT-encoding large plasmid. 


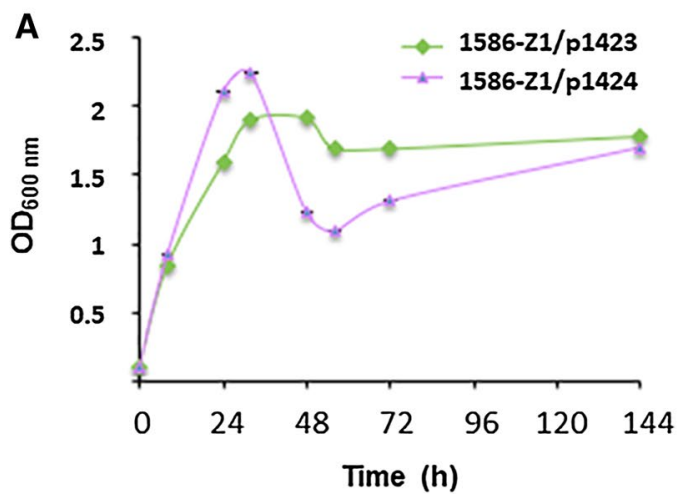

Figure 7. Growth and TeNT production in recombinant C. tetani strain 1586-Z1 (lacking the wild-type large tent plasmid), harboring the plasmid constructs p1423 (tent locus with sRNA) and p1424 (tent locus without sRNA). (A) Growth kinetics of C. tetani 1586-Z1/p1423 (containing tetR and tent genes with sRNA) and 1586Z1/p1424 (containing tetR and tent genes without sRNA) in TGY medium. (B) Extracellular TeNT levels were higher in strain 1586-Z1/p1423 than in strain 1586-Z1/p1424. Statistical significance of differences between the two recombinant strains is indicated with $p$-values $\left({ }^{\star} P<0.05 ;{ }^{* *} P<0.01\right)$. The data are from three independent experiments.

\section{Discussion}

Here, we show that the large plasmid in toxigenic $C$. tetani strains contains a conserved region downstream of the protein-coding sequence of tent. This region within or overlapping with the 3'UTR of tent encodes a sRNA of approx. 140 nucleotides that was highly expressed in the exponential growth phase. We found that this sRNA negatively regulated the expression of tent and TeNT synthesis, as judged from experiments using a RNA interference system targeting the sRNA in toxigenic clade 1A C. tetani strains. The inhibitory regulatory role of the sRNA on tent expression is likely due to sequestration of tent mRNA, since a complementary sequence of the interior junction-loop-exposed sequence of the sRNA was found in the $5^{\prime}$ end of the protein-coding part of tent (Fig. 3). It is noteworthy that the sRNA exhibited an imperfect secondary structure in clade 2 C. tetani strains (Fig. 3B) that might result in a weaker effect. The inhibitory regulatory role of the sRNA in clade $1 \mathrm{~A} C$. tetani strains is further supported by experiments showing increased levels of tent transcripts in the early exponential growth of recombinant strains containing the antisense sRNA construct as well as higher extracellular TeNT production (Figs. 5 and 6). The lower TeNT synthesis in 1586-Z1/p1424 (recombinant tetR-tent lacking the sRNA) versus in strain 1586-Z1/p1423 (tetR-tent-sRNA) is probably due to the absence of the 3'UTR of tent (51 nucleotides between the tent stop codon and sRNA) in the p1424 construction. Indeed, 3'UTRs of genes are known to contain terminator and regulatory sequences that can influence gene expression and RNA stability 27,28 . Alternatively, the apparently different results in strains lacking the sRNA (i.e. 1586-Z1/p1424 compared to the antisense strain containing p1421) might be related to the high copy number of the recombinant plasmids versus the low copy number of the wild-type large plasmid harboring tent. tent mRNA levels have not been quantified in strains 1586-Z1 and 1586-U1 transformed with p1423 or p1424.

Besides the profound impact on TeNT synthesis, the sRNA downstream of tent may be involved in the modulation of $C$. tetani growth. Altering sRNA levels in cells often lead to aberrant growth kinetics ${ }^{29,30}$. A first indication came from the antisense experiments in strain A (Fig. 6). Introducing the antisense plasmid p1421 significantly altered the growth kinetics. Further evidence came from additional experiments, e.g. the transformation of the tent locus lacking the sRNA (p1424) into the C. tetani strain 1586-Z1. This resulted in a growth pattern that is characterized by an abundant exponential growth phase followed by substantial bacterial lysis in the early stationary phase, while C. tetani strain 1586-Z1 harbouring p1423 showed a growth pattern similar to that of the wild-type strain CN655 (Fig. 7). In addition, introducing p1424 into strain CN655 resulted also in a growth pattern that was characterized by premature bacterial lysis (Sup. Fig. S4). In contrast, the transformation of p1423 (tetR-tent-sRNA) in CN655 did not modify the growth pattern of CN655 and only induced a transient over-expression of tent in the early growth phase without significant increase of extracellular TeNT (Sup. Fig. S4). These results are difficult to interpret for each individual strain, due to the simultaneous expression of recombinant tent loci with and without the sRNA (located on the plasmids p1423 and p1424) and the wild-type tent locus (located on the large plasmid). However, taken together these results indicate that the sRNA seems to contribute to the control of the growth pattern of $C$. tetani, either directly or indirectly. Indirect regulatory effects could be mediated through genes from the metabolic pathway or bacterial cell wall synthesis. The absence of sRNA in the tent locus seems to facilitate a growth pattern with an abundant exponential growth phase and premature bacterial lysis in the early stationary phase. Thus, this sRNA might have pleiotropic effects in analogy to other sRNAs. For instance, in C. perfringens, the regulatory RNA (VR-RNA) is involved in a regulatory cascade controlling 147 genes including toxin and virulence genes such as alpha-toxin, kappa-toxin, hyaluronidases, sialidase, and capsular polysaccharide synthesis ${ }^{31,32}$.

In conclusion, a sRNA lies downstream of tent and is expressed concomitantly with tent. The sRNA downregulated the expression of tent, likely by antisense sequestration of tent mRNA, and modulated the growth pattern of $C$. tetani. The interlocked activity of the sRNA regarding TeNT synthesis and bacterial growth might prevent excessive TeNT production during the exponential growth phase, thus facilitating the expansion of C. tetani. 
Unraveling the regulatory network of TeNT synthesis would allow a better understanding of the pathogenesis of tetanus as well as the in situ and in vitro production of TeNT.

\section{Materials and methods}

Plasmid and bacterial strain construction and culture conditions. C. tetani strains used in this study were: A, CN655, and 1586-Z1, a derivate of 1586-U1, but without the large tent-harboring plasmid ${ }^{22}$. Recombinant Escherichia coli BL21 strains were grown in Luria-Bertani (LB) broth, and C. tetani strains in TGY (trypticase, yeast extract, glucose) broth ( $\mathrm{pH} 7.5)$, under anaerobic conditions ${ }^{7}$. When necessary, erythromycin was added to culture media at 5 or $50 \mu \mathrm{g} / \mathrm{ml}$ for C. tetani mutants and $300 \mu \mathrm{g} / \mathrm{ml}$ for E. coli.

The pAT18 vector was used for genetic manipulation of $C$. tetani ${ }^{6}$. To investigate the effect of the putative sRNA, the sRNA-encoding DNA fragment was amplified by PCR with P2300 and P230 adding a PstI and NcoI site, respectively. The PCR-amplified sRNA was inserted in reverse orientation into pMRP306 ${ }^{26}$. The respective plasmid was named p1421 (Sup. Fig. S2). In addition, two recombinant plasmids were built: p1423 (pAT18/tetRtent including the sRNA) and p1424 (pAT18/tetR-tent without the sRNA) (Sup. Fig. S2). The primers used to PCR amplify tetR-tent-sRNA and tetR-tent are listed in Sup. Table S1. The recombinant plasmids p1421, p1423 and p1424 were transformed by electroporation into strains CN655, A, 1586-U1 and 1586-Z1. The transformants were selected on erythromycin $(5 \mu \mathrm{g} / \mathrm{ml})$ TGY agar plates.

Tetanus toxin assay. At $8,24,32,48,56,72$ and $144 \mathrm{~h}$ of growth, $4 \mathrm{ml}$ of culture were removed. The cells were harvested at 22,000 RCF for $10 \mathrm{~min}$ at $4{ }^{\circ} \mathrm{C}$, and the supernatants corresponding to the extracellular toxin were filtered $(0.22 \mu \mathrm{m})$ and stored at $-20^{\circ} \mathrm{C}$. In order to recover intracellular toxin, the pellets were washed with water and osmotic lysis was performed by homogenization and incubation in TGY containing $20 \mathrm{mg} / \mathrm{ml} \mathrm{NaCl}$ and $13.3 \mathrm{mg} / \mathrm{ml}$ sodium citrate $\left(\mathrm{C}_{6} \mathrm{H}_{5} \mathrm{Na}_{3} \mathrm{O}_{7}, 2 \mathrm{H}_{2} \mathrm{O}\right)$ during $24 \mathrm{~h}$ at $4{ }^{\circ} \mathrm{C}$. After centrifugation, the pellet was lysed again in the same procedure and same conditions. Finally, supernatants were filtered and stored at $-20^{\circ} \mathrm{C}$.

Extracellular and intracellular TeNT levels were determined by an enzyme-linked immunosorbent assay (ELISA) as previously described ${ }^{7}$.

Total RNA extraction, reverse transcription and quantitative real-time PCR analysis. Total RNA from C. tetani strains were extracted at 8, 24, 32, and $48 \mathrm{~h}$ of growth, extracted, treated with DNAse (TURBO DNA-free kit, Ambion), and converted in cDNAs as previously described ${ }^{7}$.

Real-time quantitative RT-PCR was performed in duplicate in a $25 \mu \mathrm{l}$ reaction volume containing $30 \mathrm{ng}$ of cDNAs, $12.5 \mu$ l of SYBR Green Supermix (Bio-Rad, 2X; 1,25 U iTaq DNA polymerase, $0.4 \mathrm{mM}$ each dNTP, $6 \mathrm{mM}$ $\mathrm{MgCl}_{2}, 20 \mathrm{nM}$ fluorescein, SYBR Green I) and $500 \mathrm{nM}$ gene-specific primers (Sup. Table S1) in an iQ iCycler apparatus (Bio-Rad). $r p o B$ and $g y r A$ were used as an internal reference as previously described ${ }^{7}$.

The relative cDNA quantity of each sample was determined with threshold cycle $[\Delta \Delta \mathrm{CT}]$ method $^{33,34}$. cDNA quantity of tent and tetR genes was normalized to the quantity of cDNA of the $r p o B$ and gyrA gene. Primers used were as follows: tent, forward 5'-CCAAGGTGCACAAGGAATTT-3' and reverse 5'- CAATGTTTAATGCGG GTCCT-3'; gyrA, forward 5' - AAGATGATGTAGCAGTAAGTATGGA-3' and reverse 5' - CTCTGAAGCCAA TGTCCTTTT-3'; and rpoB, forward 5'-TTGAAGAATGTAAAGAGAGAGATGCTAC-3' and reverse 5'- GGG AAGTCACCCATAAAGACA-3'.

Northern blot. Northern blot analysis was performed using North2South Chemiluminescent Hybridization and Detection Kit (Thermo Scientific) with biotin oligonucleotides probes according to manufacturer's recommendations. Briefly, total RNA samples $(5 \mu \mathrm{g})$ were run in a $10 \%$ denaturing polyacrylamide gel with $8 \mathrm{M}$ urea in $1 \times$ TBE (Tris-borate-EDTA buffer). The RNA gels were transferred to Hybond-N + (Amersham Biosciences) at $50 \mathrm{~V}$ (60-90 $\mathrm{min})$ using a Trans-Blot transfer cell (Bio-Rad). The RNA was fixed onto the membrane by a UV cross-linking. Prehybridization was carried out for $1 \mathrm{~h}$ at $55^{\circ} \mathrm{C}$ in North2South Hybridization Buffer. Hybridization was performed overnight at $55^{\circ} \mathrm{C}$ in the same buffer with biotin-labeled DNA oligonucleotide probe and membranes were washed. The size of the transcripts was estimated by comparison with RNA molecular weight standards (Invitrogen). Then, the membranes were washed twice for $5 \mathrm{~min}$ in $2 \times$ SSC ( $300 \mathrm{mM}$ sodium chloride and $30 \mathrm{mM}$ sodium citrate) $0.1 \%$ sodium dodecyl sulphate (SDS) buffer and twice for $15 \mathrm{~min}$ in $0.1 \times$ SSC $0.1 \%$ SDS buffer. Labeled probes were detected with streptavidin-HRP and the Chemiluminescent Nucleic Acid Detection Module (Thermo Scientific).

RNA seq. Total RNA was extracted as described above. The cDNA libraries were constructed by Vertis Biotechnology AG, Germany, as previously described ${ }^{35,36}$. The cDNA libraries were sequenced using a HiSeq 2000 instrument (Illumina) in a single-read mode and 100 cycles. Detailed description of procedures used for quality control, read mapping, expression graph construction and normalization of expression graphs have been published previously ${ }^{35}$. For graph visualization the Integrative Genome Browser (IGB v8.5.4) was used ${ }^{37}$.

DNA sequences are from genomes of C. tetani strain A (GenBank JWIX00000000) and C. tetani CN655 (GenBank (JSWC00000000). The RNA sequences discussed in this publication have been deposited in NCBI's Gene Express Omnibus ${ }^{38}$ and are accessible through GEO series accession number GSE150141.

Statistics. Values throughout the manuscript are expressed as means \pm standard error of the mean. Differences in the different isogenic anti-sense strains were assessed using unpaired Student's t-test where statistical significance is assumed for ${ }^{\star} \mathrm{P}<0.05,{ }^{* *} \mathrm{P}<0.01$, and ${ }^{\star *} \mathrm{P}<0.001$. 
Received: 5 August 2020; Accepted: 29 January 2021

Published online: 18 February 2021

\section{References}

1. Rossetto, O., Scorzeto, M., Megighian, A. \& Montecucco, C. Tetanus neurotoxin. Toxicon 66, 59-63. https://doi.org/10.1016/j.toxic on.2012.1012.1027 (2012).

2. Hassel, B. Tetanus: pathophysiology, treatment, and the possibility of using botulinum toxin against tetanus-induced rigidity and spasms. Toxins (Basel). 5, 73-83. https://doi.org/10.3390/toxins5010073 (2013).

3. Schmieg, N., Berscenyi, K. \& Schiavo, G. in The Comprehensive Sourcebook of Bacterial Protein Toxins (eds J. Alouf, D. Ladant, \& M. R. Popoff) Ch. 12, 337-360 (Elsevier, 2015).

4. Surana, S. et al. The travel diaries of tetanus and botulinum neurotoxins. Toxicon 147, 58-67 (2018).

5. Brüggemann, H. et al. The genome sequence of Clostridium tetani, the causative agent of tetanus disease. Proc. Natl. Acad. Sci. (USA) 100, 1316-1321 (2003).

6. Marvaud, J. C., Eisel, U., Binz, T., Niemann, H. \& Popoff, M. R. tetR is a positive regulator of the Tetanus toxin gene in Clostridium tetani and is homologous to botR. Infect. Immun. 66, 5698-5702 (1998).

7. Chapeton-Montes, D. et al. Tetanus toxin synthesis is under the control of A complex network of regulatory genes in Clostridium tetani. Toxins (Basel). 12, E328. doi:https://doi.org/10.3390/toxins12050328 (2020).

8. Brantl, S. \& Bruckner, R. Small regulatory RNAs from low-GC Gram-positive bacteria. RNA Biol. 11, 443-456. https://doi. org/10.4161/rna.28036 (2014).

9. Chen, Y., Indurthi, D. C., Jones, S. W. \& Papoutsakis, E. T. Small RNAs in the genus Clostridium. MBio 2, e00340 (2011).

10. Johansson, J. \& Cossart, P. RNA-mediated control of virulence gene expression in bacterial pathogens. Trends Microbiol. 11, 280-285 (2003).

11. Cheah, H. L. et al. Bacterial regulatory RNAs: complexity, function, and putative drug targeting. Crit. Rev. Biochem. Mol. Biol. 53, 335-355. https://doi.org/10.1080/10409238.10402018.11473330 (2018).

12. Gripenland, J. et al. RNAs: Regulators of bacterial virulence. Nat. Rev. Microbiol. 8, 857-866. https://doi.org/10.1038/nrmicro245 7 (2010).

13. Romby, P. \& Charpentier, E. An overview of RNAs with regulatory functions in gram-positive bacteria. Cell Mol. Life Sci. 67, 217-237. https://doi.org/10.1007/s00018-00009-00162-00018 (2010).

14. Chen, J., Morita, T. \& Gottesman, S. Regulation of transcription termination of small RNAs and by small RNAs: molecular mechanisms and biological functions. Front. Cell Infect. Microbiol. 9, 201. https://doi.org/10.3389/fcimb.2019.00201 (2019).

15. Bossi, L., Figueroa-Bossi, N., Bouloc, P. \& Boudvillain, M. Regulatory interplay between small RNAs and transcription termination factor Rho. Biochim. Biophys. Acta Gene Regul. Mech. 1863, 194546. https://doi.org/10.1016/j.bbagrm.192020.194546 (2020).

16. Soutourina, O. A. et al. Genome-wide identification of regulatory RNAs in the human pathogen Clostridium difficile. PLoS Genet. 9, e1003493. https://doi.org/10.1371/journal.pgen.1003493 (2013).

17. Tan, Y., Liu, J., Chen, X., Zheng, H. \& Li, F. RNA-seq-based comparative transcriptome analysis of the syngas-utilizing bacterium Clostridium ljungdahlii DSM 13528 grown autotrophically and heterotrophically. Mol. Biosyst. 9, 2775-2784. https://doi. org/10.1039/c2773mb70232d (2013).

18. Shimizu, T., Yaguchi, H., Ohtani, K., Banu, S. \& Hayashi, H. Clostridial VirR/VirS regulon involves a regulatory RNA molecule for expression of toxins. Mol. Microbiol. 43, 257-265 (2002).

19. Venkataramanan, K. P. et al. The Clostridium small RNome that responds to stress: the paradigm and importance of toxic metabolite stress in C. acetobutylicum. BMC Genomics. 14, 849. https://doi.org/10.1186/1471-2164-1114-1849 (2013).

20. Okumura, K., Ohtani, K., Hayashi, H. \& Shimizu, T. Characterization of genes regulated directly by the VirR/VirS system in Clostridium perfringens. J. Bacteriol. 190, 7719-7727 (2008).

21. Ohtani, K., Bhowmik, S. K., Hayashi, H. \& Shimizu, T. Identification of a novel locus that regulates expression of toxin genes in Clostridium perfringens. FEMS Microbiol. Lett. 209, 113-118 (2002).

22. Chapeton-Montes, D. et al. The population structure of Clostridium tetani deduced from its pan-genome. Sci. Rep. 9, 11220. https ://doi.org/10.11038/s41598-11019-47551-11224 (2019).

23. Kalvari, I. et al. Rfam 13.0: shifting to a genome-centric resource for non-coding RNA families. Nucleic Acids Res. 46, D335-D342. doi: https://doi.org/10.1093/nar/gkx1038. (2018).

24. Livny, J. \& Waldor, M. K. Identification of small RNAs in diverse bacterial species. Curr. Opin. Microbiol. 10, 96-101. https://doi. org/10.1016/j.mib.2007.1003.1005 (2007).

25. Naville, M., Ghuillot-Gaudeffroy, A., Marchais, A. \& Gautheret, D. ARNold: A web tool for the prediction of Rho-independent transcription terminators. RNA Biol. 8, 11-13. https://doi.org/10.4161/rna.4168.4161.13346 (2011).

26. Marvaud, J. C. et al. bot $\mathrm{R}$ is a positive regulator of botulinum neurotoxin and associated non toxic protein genes in Clostridium botulinum A. Mol. Microbiol. 29, 1009-1018 (1998).

27. El Mouali, Y. \& Balsalobre, C. 3'untranslated regions: regulation at the end of the road. Curr. Genet. 65, 127-131. https://doi. org/10.1007/s00294-00018-00877-x (2019)

28. Ren, G. X., Guo, X. P. \& Sun, Y. C. Regulatory 3' untranslated regions of bacterial mRNAs. Front. Microbiol. 8, 1276. https://doi. org/10.3389/fmicb.2017.01276.eCollection02017 (2017).

29. Holmqvist, E. \& Wagner, E. G. H. Impact of bacterial sRNAs in stress responses. Biochem. Soc. Trans. 45, 1203-1212. https://doi. org/10.1042/BST20160363 (2017).

30. Mars, R. A. et al. Small regulatory RNA-induced growth rate heterogeneity of Bacillus subtilis. PLoS Genet. 11, e1005046. https:// doi.org/10.1371/journal.pgen.1005046.eCollection1002015Mar (2015).

31. Ohtani, K. et al. Identification of a two-component VirR/VirS regulon in Clostridium perfringens. Anaerobe 16, 258-264 (2010).

32. 32Ohtani, K. \& Shimizu, T. Regulation of toxin production in Clostridium perfringens. Toxins (Basel) 8, 1 (2016).

33. Kirk, D. G., Palonen, E., Korkeala, H. \& Lindstrom, M. Evaluation of normalization reference genes for RT-qPCR analysis of spo0A and four sporulation sigma factor genes in Clostridium botulinum Group I strain ATCC 3502. Anaerobe 26, 14-19 (2014).

34. Livak, K. J. \& Schmittgen, T. D. Analysis of relative gene expression data using real-time quantitative PCR and the 2(-Delta Delta C(T)) Method. Methods 25, 402-408 (2001).

35. Dugar, G. et al. High-resolution transcriptome maps reveal strain-specific regulatory features of multiple Campylobacter jejuni isolates. PLoS Genet. 9, e1003495. https://doi.org/10.1371/journal.pgen.1003495 (2013).

36. Sharma, C. M. et al. The primary transcriptome of the major human pathogen Helicobacter pylori. Nature 464, 250-255. https:// doi.org/10.1038/nature08756 (2010).

37. Nicol, J. W., Helt, G. A., Blanchard, S. G. Jr., Raja, A. \& Loraine, A. E. The Integrated Genome Browser: Free software for distribution and exploration of genome-scale datasets. Bioinformatics 25, 2730-2731. https://doi.org/10.1093/bioinformatics/btp2472 (2009).

38. Edgar, R., Domrachev, M. \& Lash, A. E. Gene Expression Omnibus: NCBI gene expression and hybridization array data repository. Nucleic Acids Res. 30, 207-210. https://doi.org/10.1093/nar/1030.1091.1207 (2002). 


\section{Acknowledgements}

This work was supported by Grant from Sanofi-Pasteur and Institut Pasteur, Paris.

\section{Author contributions}

M.R.P., H.B., D.C. conceived and designed the experiments; D.C., H.B., M.R.P. performed the experiments; D.C., H.B., M.R.P. analyzed the data; L.P. contributed reagents/materials/analysis tools; M.R.P., H.B. wrote the manuscript. All authors have read and agreed to the manuscript.

\section{Competing interests}

L.P. was employed by the company Sanofi-Pasteur. The remaining authors declare that the research was conducted in the absence of any commercial or financial relationships that could be construed as a potential conflict of interest.

\section{Additional information}

Supplementary Information The online version contains supplementary material available at https://doi. org/10.1038/s41598-021-83623-0.

Correspondence and requests for materials should be addressed to M.R.P.

Reprints and permissions information is available at www.nature.com/reprints.

Publisher's note Springer Nature remains neutral with regard to jurisdictional claims in published maps and institutional affiliations.

(c) (i) Open Access This article is licensed under a Creative Commons Attribution 4.0 International License, which permits use, sharing, adaptation, distribution and reproduction in any medium or format, as long as you give appropriate credit to the original author(s) and the source, provide a link to the Creative Commons licence, and indicate if changes were made. The images or other third party material in this article are included in the article's Creative Commons licence, unless indicated otherwise in a credit line to the material. If material is not included in the article's Creative Commons licence and your intended use is not permitted by statutory regulation or exceeds the permitted use, you will need to obtain permission directly from the copyright holder. To view a copy of this licence, visit http://creativecommons.org/licenses/by/4.0/.

(C) The Author(s) 2021 